\title{
FOREWORD
}

\section{Foreword to the special issue on "the speech communication and its related technologies"}

\author{
Akinori Ito \\ Editor-in-Chief \\ Graduate School of Engineering, Tohoku University, \\ 6-6-5, Aramaki aza Aoba, Aoba-ku, Sendai, 980-8579 Japan
}

It is my great pleasure to introduce the special issue on "the speech communication and its related field." In 2011, the editorial board of the Acoustical Science and Technology decided to publish special issues periodically for further promotion of research in the acoustics, and this issue is the very first one.

Speech communication is an important field in acoustics. In addition to the human-human speech communication, speech-based human-machine communication has become popular because of popularization of smartphones and advance of cloud-based computing. The Acoustical Science and Technology has been published a number of papers in this field, and also the related discussions are made in the meetings of the Acoustical Society of Japan. In fact, more than four hundred presentations of the speech communication and related fields are made in the ASJ meeting every year. To disseminate those excellent works, we planned this special issue. The scope of this issue is all aspects of technologies related to speech communication, including speech perception and production, speech enhancement, speech coding, speech recognition and synthesis, emotion-related issues and so on.

As a result, twelve papers, one technical report and one acoustical letter have been submitted, and we accepted seven papers, one technical report and one acoustical letter. In addition, we invited two excellent reviews, both of which are related to paralinguistics, one of the most actively developing fields nowadays.

The first invited review is paralinguistics in robot communication by Prof. Kobayashi and Prof. Fujie of Waseda University. They are the pioneers of using paralingustic information in human-robot communication, and the review introduces valuable information in this field. The second invited review is written by Prof. Yoichi Yamashita of Ritsumeikan University, who has been engaged in the prosody and paralinguistics. His review gives a good overview of the field of paralinguistics from a broad perspective.

The topics of the accepted papers are widespread; signal processing and speech enhancement, speech perception and paralinguistics, speech production modeling, speech and education, speech coding, and speech synthesis. This wide variety of topics seems to show the breadth of speech-related community in ASJ.

Finally, I appreciate the effort of all the reviewers and the associate editors for their contribution to the reviewing process. I also would like to express my sincere appreciation to the Secretariat of the Acoustical Society of Japan. 